\title{
Design of wireless TCP fluid model based on cross layers
} \author{
Beijing, 100044, China \\ Beijing 100044, China \\ a)13112058@bjtu.edu.cn \\ b)yxiao@bjtu.edu.cn \\ c)wangdong@bjtu.edu.cn
}

Zhichao Zhou ${ }^{1,2 a)}$, Yang Xiao ${ }^{1,2 b)}$, and Dong Wang ${ }^{1,2 c)}$

${ }^{1}$ Institute of Information Science, Beijing Jiaotong University,

${ }^{2}$ Beijing Key Laboratory of Advanced Information Science and Network Technology,

Abstract: Channel characteristic was not considered in the past stability researches of wired network. In this letter, we propose a wireless fluid model based on cross layers to describe the performance of wireless network. In the proposed model, active queue management in the wireless access point, abnormality of wireless channel and severe packets' collisions are taken into consideration. The model enables us to analyze the wireless network with numerical approach and get an insightful understanding. Our results computed from the model show good agreement with these from simulations with network simulator.

Keywords: wireless fluid model, cross layers, stability

Classification: Network

\section{References}

[1] S. Floyd and V. Jacobson, "Random early detection gateways for congestion avoidance," IEEE/ACM Trans. Netw., vol. 1, no. 4, pp. 397-413, August 1993. DOI: $10.1109 / 90.251892$

[2] C. V. Hollot, V. Misra, D. Towsley, and W. B. Gong, "Analysis and design of controllers for AQM routers supporting TCP flows," IEEE Trans. Autom. Control, vol. 47, no. 6, pp. 945-959, June 2002. DOI:10.1109/TAC.2002.1008360

[3] L. Tan, W. Zhang, G. Peng, and G. Chen, "Stability of TCP/RED system in AQM routers," IEEE Trans. Autom. Control, vol. 51, no. 8, pp. 1393-1398, August 2006. DOI:10.1109/TAC.2006.876802

[4] G. Bianchi, "Performance analysis of the IEEE 802.11 distributed coordination function," IEEE J. Sel. Areas Commun., vol. 18, no. 3, pp. 535-547, March 2000. DOI:10.1109/49.840210

[5] B. Bellalta, M. Oliver, M. Meo, and M. Guerrero, "A simple model of the IEEE 802.11 MAC protocol with heterogeneous traffic flows," EUROCON 2005, Computer as a Tool, Belgrade, Serbia and Montenegro, no. 15, pp. 1830-1833, November 2005. DOI:10.1109/EURCON.2005.1630335

[6] Y. Xiao and M. H. Lee, "2-D Laplace-Z transformation," IEICE Trans. Fundamentals, vol. E89-A, no. 5, pp. 1500-1504, May 2006. DOI:10.1093/ietfec/e89-a. 5.1500

[7] Y. Xiao, H. Du, and Z. Cao, "2-D stability analysis for bottleneck networks with active queue management (AQM)," Chinese J. Electron., vol. 16, no. 3, pp. 519 523, July 2007. 


\section{Introduction}

Active queue management has been researched in [1, 2, 3]. In [1], the author firstly proposed the random early detection (RED) scheme and analyzed its advantages over other congestion control algorithms. In the following works [2, 3], a fluid model was established based on the RED scheme to describe the details and control congestion of network by managing the dynamic queue length of routers. The fluid model was verified available and effective. However, they assumed the channel is an ideal one. The assumption is reasonable for the far more stable wired link, but it is no longer suitable for the instable wireless network. Abnormality, packets' collisions and fading are more frequent in the wireless environment than the wired link. We take these latencies into consideration and propose a wireless TCP fluid model based on cross layers. In WFM, the elastic TCP flows are considered and the RED is applied in the wireless AP. When a packet fails three consecutive retransmissions, the MAC layer realizes severe collisions occurred or the wireless channel turned abnormal. It will inform the TCP layer of sender instantly. Thus the TCP layer of sender will halve the sending window correspondingly and recover gradually when the packet's acknowledgement arrivals.

The rest of this letter is organized as follows. In Section 2, we analyze the property of wireless channel briefly. Section 3 describes the proposed WFM. In Section 4, we validate the model with NS2. We present conclusion in Section 5.

\section{Wireless channel analysis}

Communication in the wireless channel is easily disturbed by some abnormal factors. The most considerable one is collision in the wireless channel. The characteristics of MAC layer were researched in [4], in which a two dimensional Markov model was used to analyze the details of the wireless network. The 2-D Markov model has been validated. The collision probability of a packet can be expressed as

$$
P_{k}=1-\left(1-\tau_{k}\right)^{n-1}
$$

where $\tau_{k}$ is the probability that a terminal transmits in a randomly given time slot. $n$ is the number of terminals. Using the Markov model, the probability of $\tau_{k}$ can be written as

$$
\tau_{k}=\frac{2}{1+W_{\min }+P_{k} W_{\min } \sum_{i=0}^{m-1}\left(2 P_{k}\right)^{i}}
$$

where $W_{\min }$ is the minimum backoff window, $m$ denotes the biggest backoff stage in the MAC layer. In the WFM, we consider three consecutive collisions and the probability is

$$
P_{c}=P_{k}^{3}
$$




\section{The proposed WFM}

The proposed model is described by the following nonlinear equations:

$$
\left\{\begin{array}{l}
\dot{W}=\frac{1}{R(t)}-\frac{W(t) W(t-R(t))}{2 R(t-R(t))} P_{\text {mac }}(t-R(t)) \\
\dot{q}=\frac{N(t)}{R(t)} W(t)-C
\end{array}\right.
$$

where $\dot{X}$ denotes the time-derivative. $W(t)$ denotes the expected TCP window size. $q(t)$ means expected queue length. $R(t)$ is round-trip time. $N(t)$ represents the number of flows. $C$ is defined as the capacity of the wireless AP. $P_{\operatorname{mac}}(t)$ is the sum of three consecutive collisions probability $P_{c}$ and the active dropping probability $P_{a}(t)$. The expression of $P_{a}(t)$ is

$$
P_{a}(t)= \begin{cases}0 & 0<q_{\text {ave }}<q_{\text {min }} \\ \frac{q_{\text {ave }}-q_{\text {min }}}{q_{\text {max }}-q_{\text {min }}} P_{\text {max }} & q_{\text {min }}<q_{\text {ave }}<q_{\text {max }} \\ 1 & q_{\text {max }}<q_{\text {ave }}\end{cases}
$$

where $q_{\text {ave }}$ is the average queue length. $q_{\max }$ and $q_{\min }$ are the maximum and minimum current queue length. $P_{\max }$ is the maximum active dropping probability. The equilibrium point $\left(W_{0}, q_{0}, R_{0}, P_{0}\right)$ can be expressed as follows:

$$
\left\{\begin{array}{l}
\dot{W}=0 \Rightarrow W_{0}=\frac{2}{\sqrt{P_{0}}} \\
\dot{q}=0 \Rightarrow W_{0}=\frac{R_{0} C}{N} ; R_{0}=\frac{q_{0}}{C}+T_{m a c}
\end{array}\right.
$$

where $T_{m a c}$ is the service time for a packet except for queuing time.

$$
T_{\text {mac }}=\frac{W_{\min }-1}{2\left(1-P_{k}\right)} E[\text { slot }]+\frac{P_{k}}{1-P_{k}} T_{c}+T_{p}
$$

where $E[$ slot $]$ is the average duration of a slot [5]. $T_{c}$ means the time to deal with one collision. $T_{p}$ denotes the time for successful propagation. Due to the limited space, we cannot discuss detailedly.

Linearize Eq. (4) we get

$$
\begin{aligned}
& {\left[\begin{array}{c}
\Delta \dot{W}(t) \\
\Delta \dot{q}(t)
\end{array}\right]=\left[\begin{array}{cc}
-\frac{N}{R_{0}^{2} C} & -\frac{1}{R_{0}^{2} C} \\
\frac{N}{R_{0}} & -\frac{1}{R_{0}}
\end{array}\right]\left[\begin{array}{c}
\Delta W(t) \\
\Delta q(t)
\end{array}\right]} \\
& +\left[\begin{array}{cc}
-\frac{N}{R_{0}^{2} C} & \frac{1}{R_{0}^{2} C} \\
0 & 0
\end{array}\right]\left[\begin{array}{c}
\Delta W\left(t-R_{0}\right) \\
\Delta q\left(t-R_{0}\right)
\end{array}\right] \\
& +\left[\begin{array}{c}
-\frac{R_{0} C^{2}}{2 N^{2}} \\
0
\end{array}\right] \Delta P_{m a c}\left(t-R_{0}\right)
\end{aligned}
$$

where $\Delta W(t)=W(t)-W_{0}, \Delta q(t)=q(t)-q_{0}, \Delta P_{\mathrm{mac}}(t)=P_{\mathrm{mac}}(t)-P_{0}$.

Transform Eq. (8) into Laplace domain using the approach of [6], and we obtain

$$
\left[\begin{array}{c}
\Delta W(s) \\
\Delta q(s)
\end{array}\right]=\left[s \boldsymbol{I}-A-B e^{-s R_{0}}\right]^{-1}\left[\left[\begin{array}{c}
\Delta W(0) \\
\Delta q(0)
\end{array}\right]+D \Delta P_{m a c}(s) e^{-s R_{0}}\right]
$$

where 


$$
s \boldsymbol{I}=\left[\begin{array}{ll}
S & 0 \\
0 & S
\end{array}\right], A=\left[\begin{array}{cc}
-\frac{N}{R_{0}^{2} C} & -\frac{1}{R_{0}^{2} C} \\
\frac{N}{R_{0}} & -\frac{1}{R_{0}}
\end{array}\right], B=\left[\begin{array}{cc}
-\frac{N}{R_{0}^{2} C} & \frac{1}{R_{0}^{2} C} \\
0 & 0
\end{array}\right], D=\left[\begin{array}{c}
-\frac{R_{0} C^{2}}{2 N^{2}} \\
0
\end{array}\right] .
$$

The characteristic polynomial of Eq. (9) is

$$
\begin{aligned}
B(s) & =\operatorname{det}\left[s \boldsymbol{I}-A-B e^{-s R_{0}}\right] \\
& =s^{2}+\left(\frac{N}{R_{0}^{2} C}+\frac{1}{R_{0}}+\frac{N}{R_{0}^{2} C} e^{-s R_{0}}\right) s+\frac{2 N}{R_{0}^{3} C}
\end{aligned}
$$

Let $e^{-s}=z$, we can get the 2-D Laplace-Z system of Eq. (9)

$$
\left[\begin{array}{c}
\Delta W(s, z) \\
\Delta q(s, z)
\end{array}\right]=\left[s \boldsymbol{I}-A-B z^{R_{0}}\right]^{-1}\left[\left[\begin{array}{c}
\Delta W(0) \\
\Delta q(0)
\end{array}\right]+D \Delta P_{\operatorname{mac}}(s) z^{R_{0}}\right]
$$

Then we obtain the characteristic polynomial of the 2-D Laplace-Z system in Eq. (11)

$$
\begin{aligned}
B(s, z) & =\operatorname{det}\left[s \boldsymbol{I}-A-B z^{R_{0}}\right] \\
& =s^{2}+\left(\frac{N}{R_{0}^{2} C}+\frac{1}{R_{0}}+\frac{N}{R_{0}^{2} C} z^{R_{0}}\right) s+\frac{2 N}{R_{0}^{3} C}
\end{aligned}
$$

There are two theorems about stability of $\mathrm{B}(\mathrm{s}, \mathrm{z})$ in [7]

Theorem 1: The requirement to make Eq. (10) stable, when

$$
B(s, z) \neq 0, \text { when }|z| \geq 1 \text { and } \operatorname{Re}(s) \geq 0
$$

Theorem 2: The polynomial $B(s, z)$ is stable if and only if

$$
\begin{gathered}
B(-1, z) \neq 0, \text { when }|z| \geq 1 \\
B\left(s, e^{-j w}\right) \neq 0, \text { for } w \in R, \operatorname{Re}(s) \geq 0
\end{gathered}
$$

Apply Eq. (14) to the polynomial Eq. (12), we make $B(-1, z)=0$, then get

$$
B(-1, z)=1-\left(\frac{N}{R_{0}^{2} C}+\frac{1}{R_{0}}+\frac{N}{R_{0}^{2} C} z^{R_{0}}\right)+\frac{2 N}{R_{0}^{3} C}=0
$$

From Eq. (16)

$$
z=\left(\frac{R_{0}^{3} C-R_{0} N-R_{0}^{2} C+2 N}{R_{0} N}\right)^{\frac{1}{R_{0}}}
$$

According to the converse-negative proposition of Eq. (14), if $B(-1, z)=0$, the pole $z$ has to satisfy $|z|<1$. Then we can obtain

$$
N<\frac{R_{0}^{2} C-R_{0}^{3} C}{2-2 R_{0}}
$$

From Eq. (15), we know that the symmetry axis of the quadratic is $-\left(\frac{\frac{N}{R_{0}^{2} C}+\frac{1}{R_{0}}+\frac{N}{R_{0}^{2} C} e^{-j w R_{0}}}{2}\right)<0$, and $B\left(s, e^{-j w}\right)>0$ when $\operatorname{Re}(s) \geq 0$. So Eq. (15) is tenable for the wireless network.

From the above analysis we get the ultimate scope of terminals as shown in Eq. (18). 


\section{Simulation with NS2}

We adopt a dumbbell simulation topology. The packet size is 1000 bytes, the maximum capacity between the wireless AP and the router is 1250 packets/sec, and the round-trip time is $120 \mathrm{~ms}$. The buffer size of AP is 600 packets, the minimum queue length is 60 packets and the maximum is 200 packets. The maximum active dropping probability is 0.05 . According to these parameters we can compute the reasonable number of terminals $N \in(0,10]$ with Eq. (18). We set the number to be 10 , then we obtain the instantaneous queue length in the AP and subscriber's window size from the experiments.

Fig. 1 shows the queue length from the proposed model and the instantaneous queue length from the NS2. In order to simplify the model, we do not consider the process of register, authentication, correlation between the terminals and AP. As the previous fluid model, time-out retransmission of TCP packet is also not considered. So the oscillations of the two results are different at beginning. However, during the process of stable state, the instantaneous queue length fluctuates around the model result.

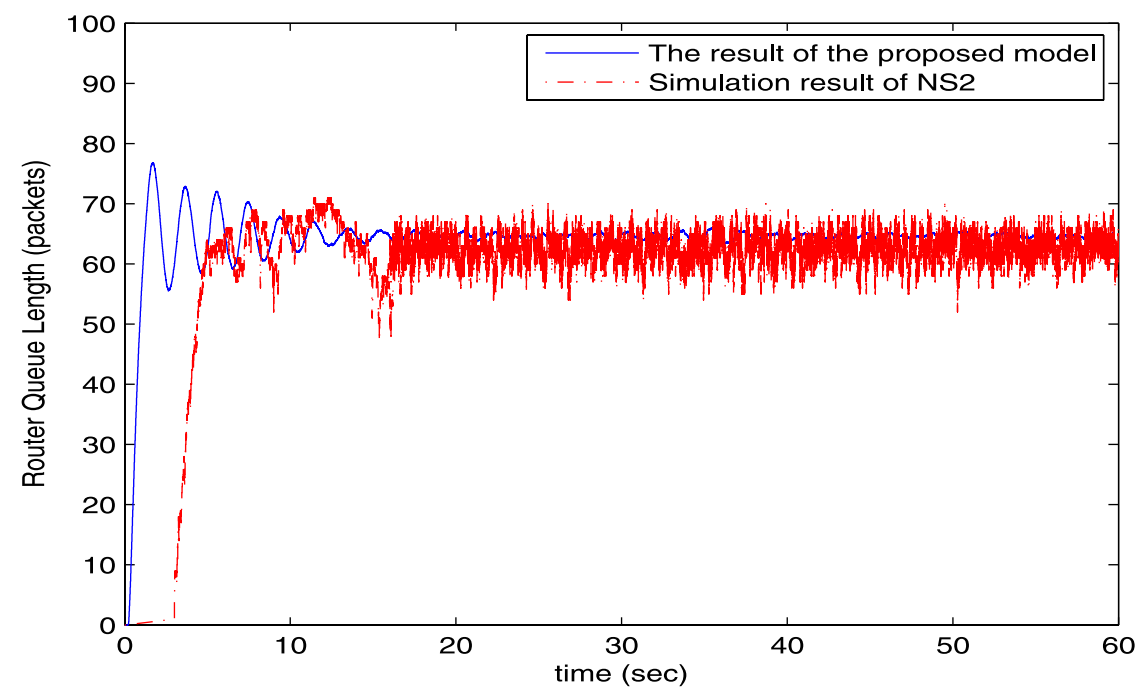

Fig. 1. Queue length evolution.

Fig. 2 illustrates the subscriber's TCP window size from the proposed model and real network simulation with NS2. The window size from NS2 is the average of the ten terminals' windows. In the stable state, the real result oscillates around the result of the model in a small scope. Fig. 1 and Fig. 2 clearly show that the proposed model results match well with the NS2 simulation in stable state, which verifies the accuracy of the proposed model. Then we set the number of terminals to be 8 and 15 to observe queue length in AP and window size of terminal. Fig. 3 shows the queue length of 15 terminals which exceed the computed scope oscillates all the way and the wireless system cannot reach a stable state. While the network is aggressive to converge to a stable state, when the subscribers are 8. Fig. 3 validates the Eq. (18). 


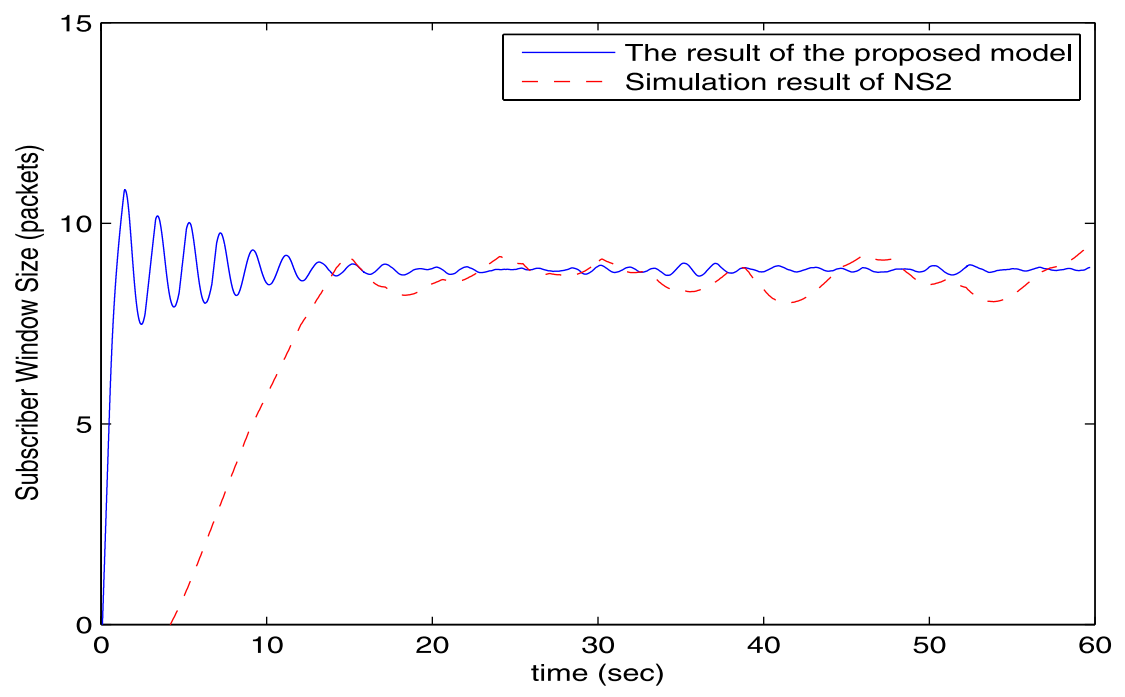

Fig. 2. Subscriber TCP window size.

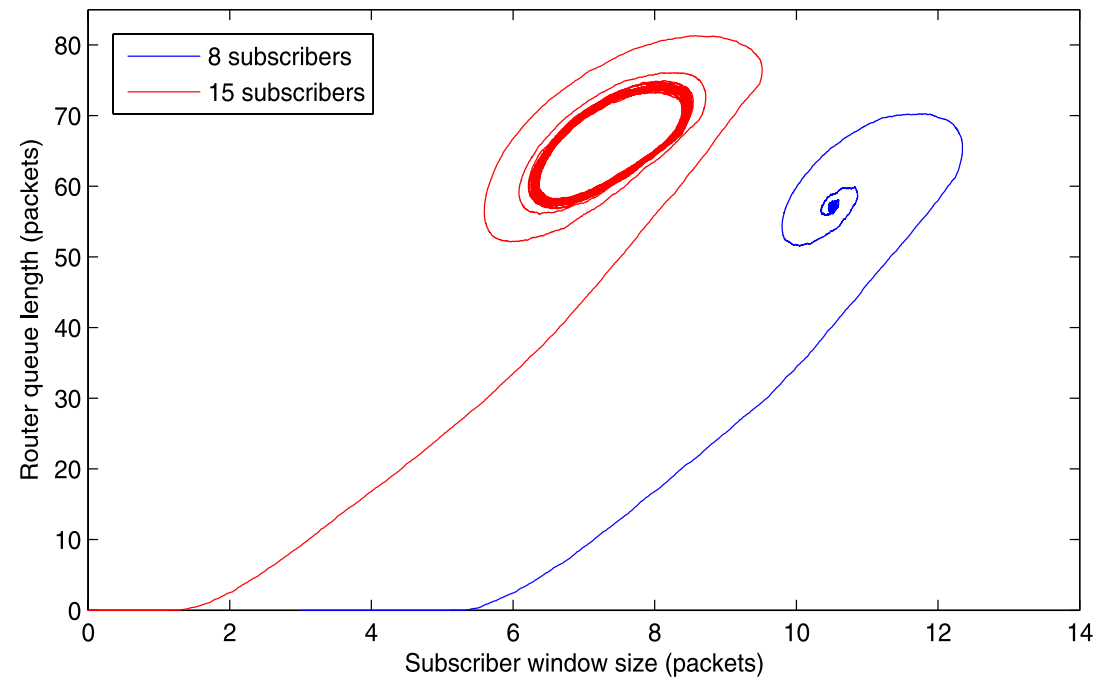

Fig. 3. Queue length and window size.

\section{Conclusion}

The former researches about the RED scheme focus on the wired network. They assume the channel is ideal, which is no longer suitable for wireless network. In this letter, we propose a fluid model based on cross layers to analyze the wireless network. We consider the packets collisions, abnormality of the wireless channel and handle the latencies with cross layers. And we can compute the scope of terminals under given bandwidth scenario with the proposed WFM. The simulations verifies the model is valid and effective.

\section{Acknowledgments}

This work is supported by the National Natural Science Foundation of China (Grant No: 61106022) and Beijing Natural Science Foundation (No: 4143066). 